\title{
Atomistic Explanation for Interlayer Charge Transfer in Metal- Semiconductor Nanocomposites: The Case of Silver and Anatase
}

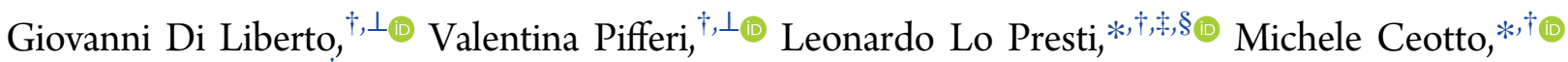 \\ and Luigi Falciola* $* \bullet$
}

\author{
${ }^{\dagger}$ Dipartimento di Chimica, Università degli Studi di Milano, Via Golgi 19, I-20133 Milano, Italy \\ ${ }^{\ddagger}$ Istituto di Scienze e Tecnologie Molecolari, Italian CNR, Via Golgi 19, I-20133 Milano, Italy \\ ${ }^{\S}$ Center for Materials Crystallography, Århus University, Langelandsgade 140, DK-8000 Århus, Denmark
}

\section{Supporting Information}

\begin{abstract}
A concerted theoretical and experimental investigation of the silver/ anatase hybrid nanocomposite, a very promising material for advanced sensing applications, is presented. We measure its exceptional electrochemical virtues in terms of current densities and reproducibility, providing their explanation at the atomic-scale level and demonstrating how and why silver acts as a positive electrode. Using periodic plane-wave DFT calculations, we estimate the overall amount of electron transfer toward the semiconductor side of the interface at equilibrium. Suitably designed (photo)electrochemical experiments strictly agree, both qualitatively and quantitatively, with the theoretical charge transfer estimates. The unique permanent charge separation occurring in the device is possible because of the favorable synergy of $\mathrm{Ag}$ and $\mathrm{TiO}_{2}$, which exploits in a favorable band alignment, while the electron-hole recombination rate and carrier mobility decrease when electrons cross the metal-semiconductor interface. Finally, the hybrid material is proven to be extremely robust against aging, showing complete regeneration, even after 1 year.

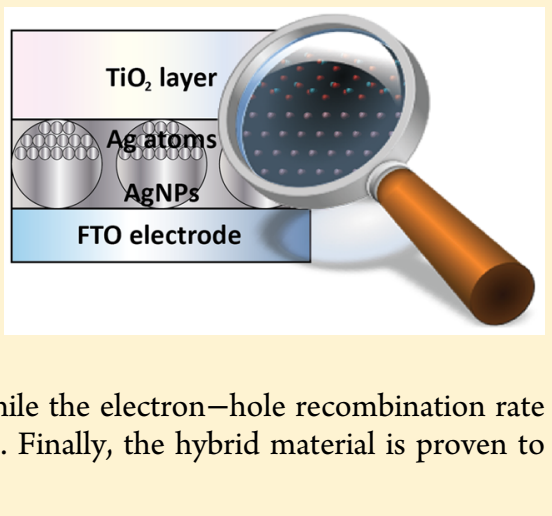

$\mathrm{T}$ he use of hybrid nanomaterials, characterized by unprecedented behaviors and features, has now paved the way toward promising applications in many fields, ${ }^{1-3}$ such as electrocatalysis, photocatalysis, electroanalysis, and environmental chemistry. Recently, Serpone et al. ${ }^{4}$ suggested that such a third generation of photoactive devices has the potential to significantly impact everyday life. Suitably designed nanoheterojunctions enhance synergistic functionalities and allow one to obtain "brave new materials" with physicochemical properties that are not simply the addition of the precursors' ones ${ }^{4}$ but are completely new, different, and unexpected. ${ }^{5,6}$ However, research on such devices is most often dominated by trial and error procedures, while a deep atomistic understanding of the phenomena inside of the junction region driving appropriate design of the final device is missing.

A central and key nanocomposite is $\mathrm{Ag}-\mathrm{TiO}_{2},{ }^{7,8}$ which has been already used for several photocatalytic purposes ${ }^{9,10}$ and more recently engineered for the electroanalytical determination of relevant neurotrasmitters. ${ }^{11}$ It provided the first photorenewable sensor device, pushing the limits in terms of accuracy, sensitivity, detection limits, and photoactivity. ${ }^{12}$ Considering that $\mathrm{TiO}_{2}$ semiconductors are usually useless in electroanalytical applications and $\mathrm{Ag}$ is subject to fouling and oxidation/passivation, such broad outcomes were totally unexpected. Despite the ongoing research, ${ }^{13,14}$ a quantitative and comprehensive understanding of the physics behind this nanocomposite is still missing, thus preventing its full exploitation and the extension of the same paradigm to other systems and devices. We here aim at explaining the electronic charge events occurring at the silver/titania interface through a concerted theoretical and experimental multidisciplinary approach.

Ag nanoparticles (NPs) are immobilized on a fluorine-doped tin oxide (FTO) support ${ }^{15}$ and are covered with a thin anatase $\mathrm{TiO}_{2}$ layer ${ }^{16}$ (full details on the synthetic procedure are in SI section S1). The properties of three different FTO electrodes, prepared with different covering material, were compared: (A) $\mathrm{Ag}$ NPs, (B) composite Ag-TiO 2 NPs (Figure S1-1a), and (C) $\mathrm{TiO}_{2}$ NPs. The first striking difference of the hybrid Ag-TiO system with respect to the other ones is evident from cyclic voltammetry $(\mathrm{CV})$ in $0.1 \mathrm{M} \mathrm{NaClO}_{4}$ (Figure 1a). The Agcovered electrode (A, red line, Figure 1a) presents the typical plateau at positive potentials associated with the formation of Ag oxides on the metallic Ag surface, which is regenerated in the reduction region, ${ }^{17-20}$ with compatible peak areas. As expected, no faradaic current is detected for the large-gap $\mathrm{TiO}_{2}$ semiconductor NPs (C, green line, Figure 1a). Instead, the Ag$\mathrm{TiO}_{2}$ voltammogram (B, blue line, Figure 1a) presents an unexpected very sharp oxidation peak, shifted to a more favorable potential, with an area two times higher than that of the reduction counterpart, even though the quantity and type of silver deposited is the same for both the $\mathrm{A}$ and $\mathrm{C}$ modified electrodes. To gain insights into this evidence, the hetero-

Received: September 27, 2017

Accepted: October 19, 2017

Published: October 19, 2017 

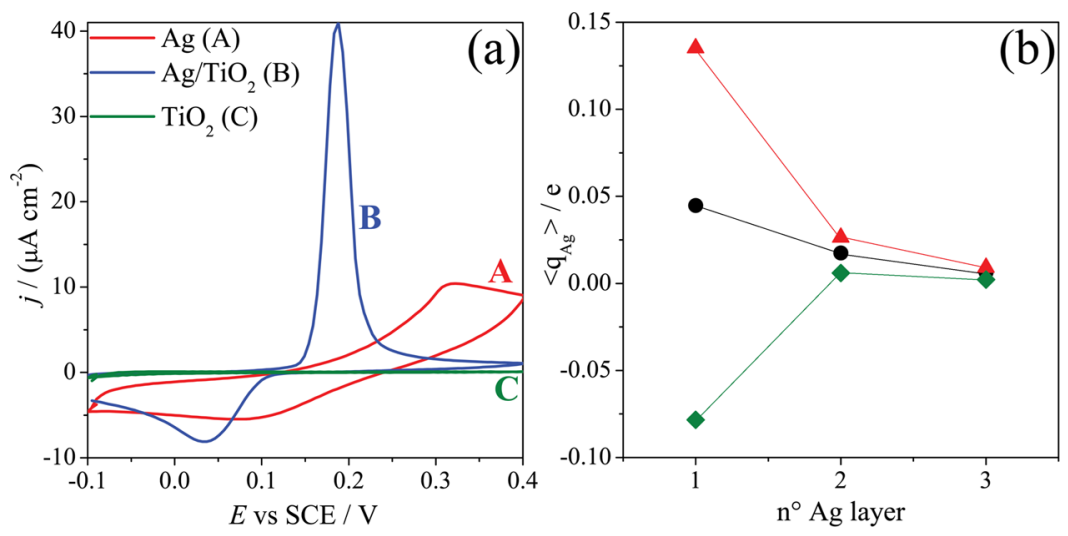

Figure 1. (a) Cyclic voltammograms $\left(0.1 \mathrm{~V} \mathrm{~s}^{-1}\right)$ for the three modified FTO electrodes. (b) Averaged QTAIM (Bader) charge (black circles; see the text and SI section S2) of $\mathrm{Ag}$ atoms in a series of layers parallel to the $\mathrm{Ag}-\mathrm{TiO}_{2}$ interface (no. 1 is the closest one). Red triangles (green diamonds) plot the most positive (negative) charges found on $\mathrm{Ag}$ anywhere in the same layer.

junction has been investigated using a PW-DFT+U theoretical approach $^{21,22}$ with a U Hubbard splitting parameter set to 3.3 $\mathrm{eV}$, in agreement with previous works on anatase. ${ }^{23-27}$ The VASP suite of codes ${ }^{21,22,28}$ was employed throughout. To build a least-biased model for the interface, $\mathrm{Ag}$ atoms were added one by one on the top surface of a previously optimized (101) anatase, ${ }^{24}$ known to be the most stable one ${ }^{29,30}$ and thus frequently exposed in NPs grown under thermodynamic control. A bulk oxygen vacancy was also explicitly considered in the $\mathrm{TiO}_{2}$ phase due to the high defective nature of synthetic $\mathrm{TiO}_{2}$ materials. ${ }^{31}$ Upon each $\mathrm{Ag}$ addition, the whole system was fully relaxed, with the only exception of the deepest $\mathrm{TiO}_{2}$ layer (i.e., the most distant from the surface), which was kept frozen at the undistorted bulk $\mathrm{TiO}_{2}$ structure. Eventually, five full $\mathrm{Ag}$ layers were grown onto the anatase (101) surface (Figure S1-1b SI). More details on the whole procedure can be found in the SI (section S2 and Figure S2-1-3). No appreciable reconstructions affect the $\mathrm{TiO}_{2}(101)$ surface model, with mesh translation modules $a_{1}$ and $a_{2}$ undergoing changes no larger than $\pm 0.1 \AA$ upon going from the pristine clean surface to that of the composite junction (Table S2-1). Ag atoms eventually achieve a fcc packing comparable to that of the native metal (space group $F m \overline{3} m, n^{\circ} 225, a=4.085(1) \AA^{32}$ ) and expose the highly stable (111) face ${ }^{33}$ at the interface. It must be stressed that neither geometry nor symmetry constraints are imposed while building the $\mathrm{Ag}$ phase; therefore, the final packing is determined just by the metal selfassembling.

The whole metal-semiconductor interface rearranges to make the surface translation vectors, $a_{1}$ and $a_{2}$, almost exactly commensurate to each other $\left(3 a_{1}\left[\mathrm{TiO}_{2}\right] / 4 a_{1}[\mathrm{Ag}]=1.0006\right.$, $\left.2 a_{2}\left[\mathrm{TiO}_{2}\right] / 5 a_{2}[\mathrm{Ag}]=1.0170\right)$ along the two periodic directions. At the interface, distances among $\mathrm{Ag}$ and surfacial $\mathrm{O}_{\text {surf }}$ atoms lie in the range of 2.4-2.8 $\AA$, slightly longer than the $\mathrm{Ag}-\mathrm{O}$ ones in $\mathrm{Ag}_{2} \mathrm{O}$ (2.1 $\AA$ ). On the other hand, $\mathrm{Ag}-\mathrm{Ag}$ distances approach the typical bulk values $(\sim 2.90 \AA)$ for layers far from the surface.

The wave function-derived total charge density, $\rho(\mathbf{r})$, allows one to go a step further with respect to the pure geometric analysis. According to the Quantum Theory of Atoms in Molecules (QTAIM), ${ }^{34}$ regions bounded by zero-flux surfaces of the gradient vector field of $\rho, \nabla \rho(\mathbf{r})$, unequivocally partition the space into atomic domains, $\Omega$. This allows a straightforward definition of atomic charges as $q_{\Omega}=Z_{\Omega}-\int_{\Omega} \rho(\mathbf{r}) \mathrm{d} \mathbf{r}, Z_{\Omega}$ being the corresponding positive nuclear charge. ${ }^{35}$ Figure $1 \mathrm{~b}$ shows $\left\langle q_{\mathrm{Ag}}\right\rangle$ estimates, i.e., layer-by-layer averaged $\mathrm{Ag}$ charges upon going from the interface $(n=1)$ to the bulk phase $(n \geq 3)$. The results are consistent with a neat electron transfer of $9.5 \mu \mathrm{C}$ $\mathrm{cm}^{-2}$ from the metal to the semiconductor. This estimate was derived from the Bader integrated charges of the $\mathrm{Ag}$ atoms at the interface (first layer) because their sum is equivalent to the number of electrons donated to the $\mathrm{TiO}_{2}$ phase. To obtain the specific charge transfer, we divided the total net charge on interfacial $\mathrm{Ag}$ atoms by the total surface of the slab. By looking at how the donated charge is distributed over the atomic sites of the semiconductor, it comes out that most of the transferred charge is located at the exposed oxygen atoms of the anatase surface, leading to an enhanced ionic behavior of the slab. However, a non-negligible fraction of the donated charge $(18 \%)$ is located at titanium sites, modulating its electronwithdrawing power and implying a formal partial reduction of $\mathrm{Ti}(\mathrm{IV})$ in the neighborhood $(d \leq 3 \AA)$ of the interface. On average, $\left\langle q_{\mathrm{Ti}}\right\rangle$ of the most exposed $\mathrm{Ti}$ layer decreases from +2.000 (6) lel in the isolated anatase slab to $1.968(2)$ lel in the composite $\mathrm{TiO}_{2} / \mathrm{Ag}$ system. On the contrary, Ag atoms in close contact with the interfacial $\mathrm{O}_{\text {surf }}$ atoms are neatly positive (Figure $1 \mathrm{~b}$, red triangles), ${ }^{36}$ while the other ones are neutral or slightly negative (Figure $1 \mathrm{~b}$, green diamonds). In any case, the magnitude of the charge transfer quickly fades away at increasing distances from the interface. The bulk properties of the metallic phase prevail from the fourth layer, $\sim 7 \AA$ apart from the interface. The effect of possible surface oxygen defects, though less favorable on anatase (101), ${ }^{37}$ imply just a slight reduction $(-18 \%)$ of the overall charge transfer, which

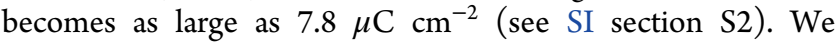
also tested the robustness of these proposed theoretical predictions against a strongly different model for the $\mathrm{TiO}_{2} /$ Ag interface, ${ }^{14}$ where anatase $(001)$ is used as a crystallization seed for building the metallic phase. The (001) face is much less stable and bears very different properties. ${ }^{29,30,38-40}$ However, also in this case, a stable interface is obtained, implying a charge transfer of $9.4 \mu \mathrm{C} \mathrm{cm}{ }^{-2}$ from $\mathrm{Ag}$ to $\mathrm{TiO}_{2}$, very close to the $(101) /(111)$ estimate of $9.5 \mu \mathrm{C} \mathrm{cm} \mathrm{cm}^{-2}$. We can thus safely conclude that our predictions hold true irrespective of the specific interface model chosen to simulate the $\mathrm{TiO}_{2}-\mathrm{Ag}$ junction. A full discussion can be found in SI section S2. Here, we remark that, even though the scenario is more complex in real samples (see below), an electron transfer process across the metal-semiconductor junction is invariably predicted to occur, 

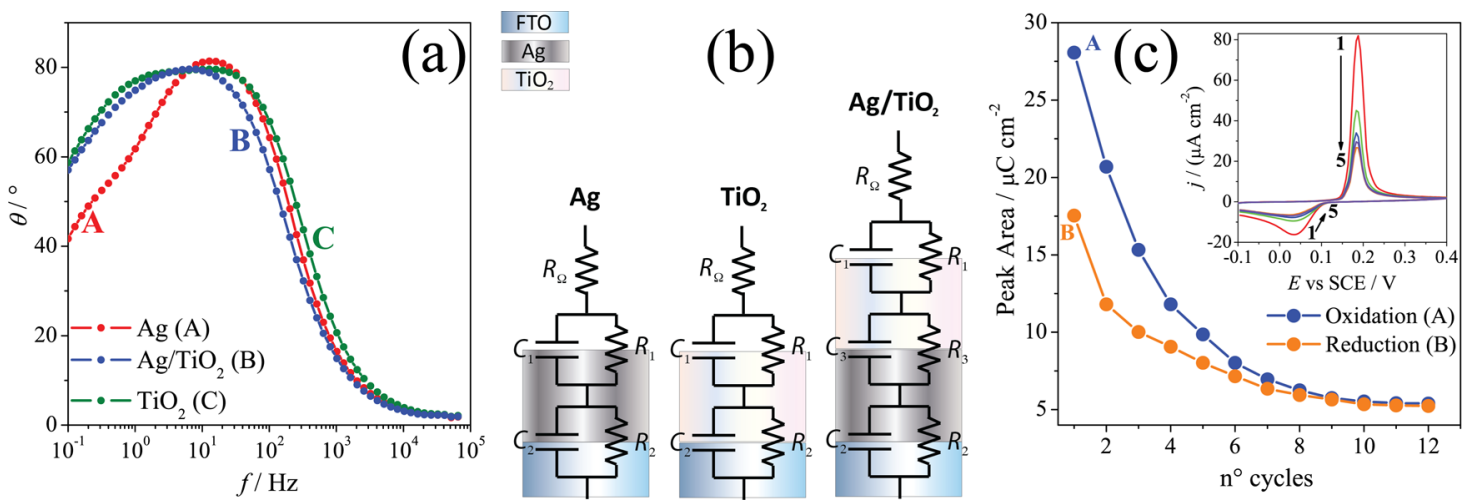

Figure 2. (a) Bode plots at $-0.1 \mathrm{~V}$ and (b) corresponding equivalent circuits of the three tested electrodes. (c) Decay of oxidation and reduction peak areas for consecutive voltammetric cycles. Inset: cyclic voltammograms of $\mathrm{Ag}-\mathrm{TiO}_{2}$ for different consecutive cycles.
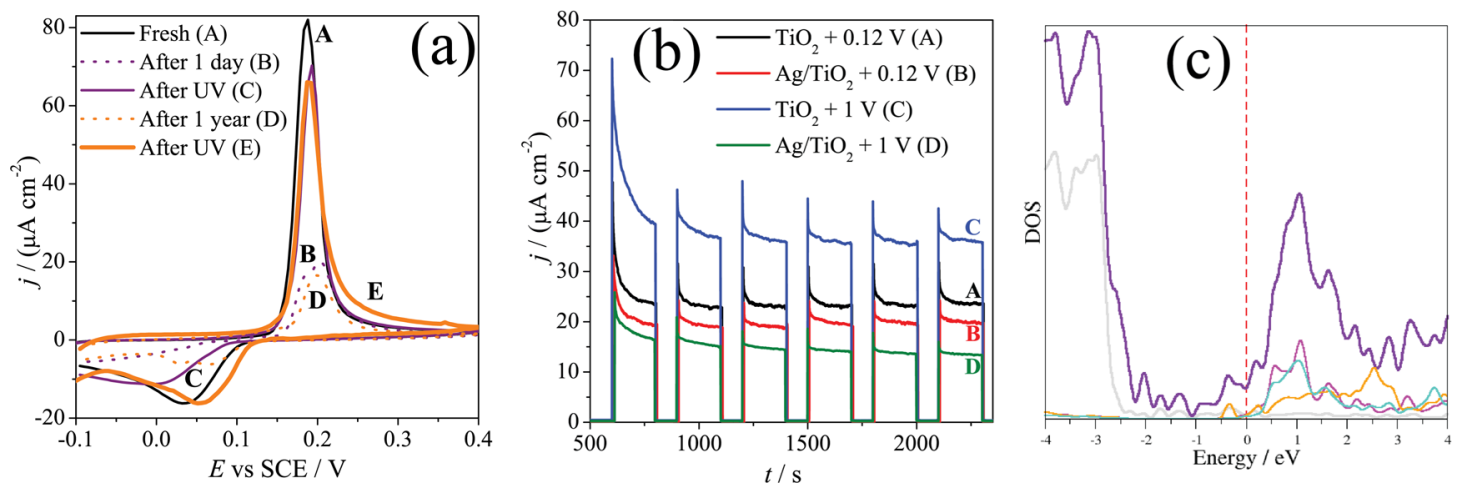

Figure 3. (a) Cyclic voltammograms of the $\mathrm{Ag}-\mathrm{TiO}_{2}$ electrode freshly prepared, after 1 day, or after 1 year of storage. CVs of the rested electrode, before UV recovery in dotted lines; (b) photocurrent on the $\mathrm{Ag}$ and $\mathrm{Ag}-\mathrm{TiO}_{2}$ electrodes with a UV iron halogenide lamp emitting in the 280-400 $\mathrm{nm}$ range with an effective power density of $23 \mathrm{~mW} \mathrm{~cm}{ }^{-2}$; and (c) computed DOS of the $\mathrm{Ag}-\mathrm{TiO}_{2}$ optimized structure (violet line) relative to the Fermi energy $(E=0 \mathrm{eV})$. Other curves represent the atom-projected partial DOS (PDOS). Gray: silver atoms; magenta: Ti atoms of the first layer, the one closest to Ag; turquoise: inner layer of $\mathrm{Ti}$ atoms; orange: $\mathrm{Ti}$ atoms closest to the bulk oxygen vacancy.

where the metal spontaneously oxidizes and leads to an increase of partially reduced $\mathrm{Ti}$ centers in the semiconductor phase.

Theoretical predictions are also supported by electrochemical impedance spectroscopy (EIS) data, whose interpretation and fitting were performed using equivalent circuits (Figure 2b) designed according to the theoretical outcomes. Complete results are reported in Table S3-1 (SI section S3). Bode (Figure 2a) and complex plane plots (SI, Figure S3-1) show that the $\mathrm{Ag}-\mathrm{TiO}_{2}$ profile is more similar to that of titania, suggesting that silver adapts to titania, in agreement with DFT calculations. The measured $R_{1}$ and $R_{2}$ resistance values for the $\mathrm{Ag}-\mathrm{TiO}_{2}$ device lie between those determined for $\mathrm{Ag}$ and $\mathrm{TiO}_{2}$, while the $\mathrm{Ag}-\mathrm{TiO}_{2}$ interface resistance $\left(R_{3}\right)$ presents lower values, suggesting a charge transfer characterized by good conduction due to the close contact of the two materials. Finally, high capacitance values (SI, Table S3-1) of the $\mathrm{Ag}-\mathrm{TiO}_{2}$ interface confirm the short-range character of the interaction region, as predicted by DFT calculations in Figure $1 \mathrm{~b}$.

The combined theoretical and impedance spectroscopy outcomes aid in providing an explanation of the voltammetric findings. In the hybrid material, $\mathrm{Ag}$ atoms closer to the interface are oxidized during the anodic scan, becoming positive ions stabilized by the presence of $\mathrm{TiO}_{2}$ and increasing the width of the charged metal region. This process, more favorable than the formation of the oxide, ${ }^{41}$ shifts the oxidation peak potential toward more favorable values. Moreover, because no insulating oxide is involved, electrons can move faster and a sharp peak instead of a plateau is registered. The reduction peak at $+0.04 \mathrm{~V}$ (vs SCE, Figure 1a) can be instead associated with the reduction of $\mathrm{Ag}^{+}$ions and to the consequent narrowing of the charged metal region. In this case, the peak potential is less favorable with respect to the $\mathrm{Ag}$ oxide dissolution peak, indicating higher stability of the positive Ag ions. ${ }^{42}$ The smaller area of the reduction peak, at least 2 times lower than the oxidation one, suggests that only part of positive Ag ions return to a metallic state. For sequential cycles, the oxidation and reduction peaks decrease rapidly and reach asymptotically a similar value (Figure 2c). This implies that a dynamic equilibrium is set up, involving the part of the metal layer far from the interface, which keeps oxidizing and reducing itself. The decrease of the peaks has been also attributed to the dissolution (stripping) of silver during the voltammetric cycling, ${ }^{18,19}$ but in our case, Ag NPs are entrapped between the FTO support and the titania layer, being thus prevented from diffusion, aggregation, and coalescence. This complies well with the regenerating photochemical properties discussed later. On the other hand, the part of Ag layer closer to titania remains oxidized. Therefore, as theoretically predicted, the composite device presents a partial positive charge, which is due to $\mathrm{Ag}^{+}$ ions close to the interface and is fixed during the voltammetric cycling. In other words, a sort of nanocomposite "positive silver electrode" is created. The transferred charge, as estimated from the areas under the voltammetric peaks (see SI section S3), amounts to $110 \mu \mathrm{C} \mathrm{cm}^{-2}$. The corresponding theoretical limits 
for the total transferable charge, representing the maximum charge that can be extracted from the interface Ag layer (see SI section S2), are comparable but slightly higher, namely, 203 and $185 \mu \mathrm{C} \mathrm{cm}^{-2}$ for the anatase (101) and (001) models. Considering the unavoidable charge dispersion in real systems and approximations in quantum models, experiment and theory provide a remarkable conformity of views, even bearing in mind that the peak currents of independent silver NPs are more than 1 order of magnitude lower. Once again, this suggests that the charge transfer responsible for the $\mathrm{CV}$ unexpected behavior is located at the interface.

Regenerating properties of the device due to the $\mathrm{TiO}_{2}$ photoactivity yield interesting electroanalytical advantages in sensor applications. ${ }^{11,12}$ This can be explained in terms of our "positive silver electrode" model. Irradiating with UV-A light, electrons transferring from the $\mathrm{TiO}_{2}$ valence band (VB) to the conduction band (CB) are localized on $\mathrm{Ag}^{+}$ions, causing their reduction to the metallic state and restoring the initial thickness of the uncharged metal. This is mirrored (i) in the complete regeneration of the sharp oxidation peak in $\mathrm{CV}$ experiments with current densities comparable with those of the freshly prepared device (Figure 3a) and (ii) in the photocurrent findings (Figure $3 \mathrm{~b}$ ). Figure $3 \mathrm{a}$ shows that the composite electrode provides very robust regenerating performances compared to bare Ag NPs. Actually, it restores its original properties even after a very long ( 1 year) rest time (Figure 3a and SI section S3). Specifically, the amount of charge transferred from $\mathrm{TiO}_{2}$ to $\mathrm{Ag}$ depends on the quantity of $\mathrm{Ag}^{+}$ to be regenerated. Working at $+0.12 \mathrm{~V}$, where $\mathrm{Ag}^{0}$ is preserved, the photocurrent values presented by $\mathrm{TiO}_{2}$ and $\mathrm{Ag}-\mathrm{TiO}_{2}$ systems are comparable (black and red curves in Figure $3 \mathrm{~b}$ ), slightly lower in the $\mathrm{Ag}-\mathrm{TiO}_{2}$ case, because a small quantity of photoelectrons are probably used to reduce naturally oxidized $\mathrm{Ag}$ atoms (foreseen by theoretical calculations) while the others can efficiently close the electrochemical circuit contributing to the measured photocurrent. Setting the potential at $+1 \mathrm{~V}$, where $\mathrm{Ag}$ atoms are also electrochemically oxidized in a large quantity and stabilized by $\mathrm{TiO}_{2}$, a significant number of photoelectrons are used for $\mathrm{Ag}^{0}$ regeneration. This produces in the nanocomposite a lower value of photocurrent (green curve in Figure 3b) with respect to $\mathrm{TiO}_{2}$ (blue curve in Figure 3b). Moreover, further evidence is provided by the increase in the electron-hole recombination time (i.e., less probable charge recombination) for the composite system as the metal acts as an electron scavenger toward the charge carriers coming from anatase (see also SI section S3). The described regenerating phenomenon is due to the materials' intimate contact, which leads to the density of states (DOS) reported in Figure 3c. The $\mathrm{VB}$ and $\mathrm{CB}$ arising from $\mathrm{TiO}_{2}$ are clearly recognizable (compare magenta, turquoise, and orange lines with the violet one). Ag states mainly contribute in the VB region, while they are less dense in the band gap. ${ }^{14}$ The tiny enhanced formal $\mathrm{Ti}(\mathrm{III})$-like behavior of exposed titanium atoms (magenta line) is also appreciable as their contribution to the total DOS very slightly shifts from that due to formal $\mathrm{Ti}(\mathrm{IV})$ ions of bulk anatase (turquoise line). On the other hand, bulk oxygen vacancies produce the expected typical shallow midgap states close to the CB (orange line). ${ }^{31,43}$

In conclusion, a synergistic electrochemical and quantum investigation of the $\mathrm{Ag}-\mathrm{TiO}_{2}$ nanocomposite electrode shed light on the peculiar electronic properties of the heterojunction region, related to the intimate contact of $\mathrm{Ag} \mathrm{NPs}$ with $\mathrm{TiO}_{2}$. A solid explanatory model, able to account for several unexpected (photo)electrochemical findings, has been developed and used to elucidate how and why the $\mathrm{Ag}-\mathrm{TiO}_{2}$ device bears unique emerging physicochemical properties. A quantitative agreement between theory and experiment in terms of charge transfer toward the semiconductor strongly supports the present interpretative framework. Future developments see exploitation of the unique virtues of the $\mathrm{Ag}-\mathrm{TiO}_{2}$ electrode for electroanalytical determination and photocatalytic removal of emerging contaminants in the liquid and gas phases. Engineering of the nanocomposite will be aimed at enhancing its sensitivity to solar light, e.g., by providing proper bulk dopants in the $\mathrm{TiO}_{2}$ phase. ${ }^{27,44}$ This also will pave the way to the application of experimental and theoretical spectroscopy tools ${ }^{44}$ for identification of the interaction mechanism of the contaminants with the device.

Finally, this approach might be applied to other cases where the heterojunction contribution is crucial. Previous studies already observed that charge transfer could take place across $\mathrm{Ag}-\mathrm{TiO}_{2}$ interfaces, but it was believed to provide negligible contributions to any measurable property. This is clearly not the case here. Our strict and quantitative comparison between experiment and theory can in principle be employed to reveal whether novel hybrid nanocomposites might bear macroscopic innovative physical properties.

\section{ASSOCIATED CONTENT}

\section{S Supporting Information}

The Supporting Information is available free of charge on the ACS Publications website at DOI: 10.1021/acs.jpclett.7b02555.

Electrode preparation; computational setup and comments on the various quantum models; and electrochemical characterization (CV, EIS, photocurrent) (PDF)

\section{AUTHOR INFORMATION}

\section{Corresponding Authors}

*E-mail: leonardo.lopresti@unimi.it (L.L.P.).

*E-mail: michele.ceotto@unimi.it (M.C.).

*E-mail: luigi.falciola@unimi.it (L.F.).

\section{ORCID}

Giovanni Di Liberto: 0000-0003-4289-2732

Valentina Pifferi: 0000-0001-7439-1022

Leonardo Lo Presti: 0000-0001-6361-477X

Michele Ceotto: 0000-0002-8270-3409

Luigi Falciola: 0000-0002-2031-239X

\section{Author Contributions}

${ }^{\perp}$ G.D.L. and V.P. contributed equally to this work.

\section{Notes}

The authors declare no competing financial interest.

\section{ACKNOWLEDGMENTS}

M.C. and G.D.L. acknowledge financial support from the European Research Council (ERC) under the European Union's Horizon 2020 research and innovation programme (Grant Agreement No. [647107] - SEMICOMPLEX - ERC2014-CoG). We acknowledge the CINECA and the Regione Lombardia award under the LISA initiative 2016-2018 project GREENTi - for the availability of high-performance computing resources and support. 


\section{REFERENCES}

(1) Wang, H.; Zhang, L.; Chen, Z.; Hu, J.; Li, S.; Wang, Z.; Liu, J.; Wang, X. Semiconductor Heterojunction Photocatalysts: Design, Construction, and Photocatalytic Performances. Chem. Soc. Rev. 2014, 43 (15), 5234.

(2) Mehdi, A.; Reye, C.; Corriu, R. From Molecular Chemistry to Hybrid Nanomaterials. Design and Functionalization. Chem. Soc. Rev. 2011, 40 (2), 563-574.

(3) Saavedra, J.; Doan, H. a.; Pursell, C. J.; Grabow, L. C.; Chandler, B. D. The Critical Role of Water at the Gold-Titania Interface in Catalytic CO Oxidation. Science 2014, 345 (6204), 1599-1602.

(4) Emeline, A. V.; Kuznetsov, V. N.; Ryabchuk, V. K.; Serpone, N. On the Way to the Creation of next Generation Photoactive Materials. Environ. Sci. Pollut. Res. 2012, 19 (9), 3666-3675.

(5) Green, I. X.; Tang, W.; Neurock, M.; Yates, J. T. Spectroscopic Observation of Dual Catalytic Sites During Oxidation of CO on a Au/ $\mathrm{TiO}_{2}$ Catalyst. Science 2011, 333 (6043), 736-739.

(6) Wang, H.; An, T.; Selloni, A. Effect of Reducible Oxide-metal Cluster Charge Transfer on the Structure and Reactivity of Adsorbed $\mathrm{Au}$ and Pt Atoms and Clusters on Anatase $\mathrm{TiO}_{2}$. J. Chem. Phys. 2017, 146 (18), 184703.

(7) Hirakawa, T.; Kamat, P. V. Photoinduced Electron Storage and Surface Plasmon Modulation in $\mathrm{Ag} @ \mathrm{TiO}_{2}$ Clusters. Langmuir 2004, 20 (14), 5645-5647.

(8) Zhang, H.; Wang, G.; Chen, D.; Lv, X.; Li, J. Tuning Photoelectrochemical Performances of $\mathrm{Ag}-\mathrm{TiO}_{2}$ Nanocomposites via Reduction/Oxidation of Ag. Chem. Mater. 2008, 20 (20), 65436549.

(9) Lu, Q.; Lu, Z.; Lu, Y.; Lv, L.; Ning, Y.; Yu, H.; Hou, Y.; Yin, Y. Photocatalytic Synthesis and Photovoltaic Application of $\mathrm{Ag}-\mathrm{TiO}_{2}$ Nanorod Composites. Nano Lett. 2013, 13 (11), 5698-5702.

(10) Hirakawa, T.; Kamat, P. V. Charge Separation and Catalytic Activity of $\mathrm{Ag} @ \mathrm{TiO}_{2}$ Core-Shell Composite Clusters under UVIrradiation. J. Am. Chem. Soc. 2005, 127 (11), 3928-3934.

(11) Soliveri, G.; Pifferi, V.; Panzarasa, G.; Ardizzone, S.; Cappelletti, G.; Meroni, D.; Sparnacci, K.; Falciola, L. Self-Cleaning Properties in Engineered Sensors for Dopamine Electroanalytical Detection. Analyst 2015, 140 (5), 1486-1494.

(12) Pifferi, V.; Soliveri, G.; Panzarasa, G.; Cappelletti, G.; Meroni, D.; Falciola, L. Photo-Renewable Electroanalytical Sensor for Neurotransmitters Detection in Body Fluid Mimics. Anal. Bioanal. Chem. 2016, 408 (26), 7339-7349.

(13) Pifferi, V.; Marona, V.; Longhi, M.; Falciola, L. Characterization of Polymer Stabilized Silver Nanoparticles Modified Glassy Carbon Electrodes for Electroanalytical Applications. Electrochim. Acta 2013, 109, 447-453.

(14) Prada, S.; Rosa, M.; Giordano, L.; Di Valentin, C.; Pacchioni, G. Density Functional Theory Study of $\mathrm{TiO}_{2} / \mathrm{Ag}$ Interfaces and Their Role in Memristor Devices. Phys. Rev. B: Condens. Matter Mater. Phys. 2011, 83 (24), 245314.

(15) Meroni, D.; Lo Presti, L.; Di Liberto, G.; Ceotto, M.; Acres, R. G.; Prince, K. C.; Bellani, R; Soliveri, G.; Ardizzone, S. A Close Look at the Structure of the $\mathrm{TiO}_{2}$-APTES Interface in Hybrid Nanomaterials and Its Degradation Pathway: An Experimental and Theoretical Study. J. Phys. Chem. C 2017, 121 (1), 430-440.

(16) Maino, G.; Meroni, D.; Pifferi, V.; Falciola, L.; Soliveri, G.; Cappelletti, G.; Ardizzone, S. Electrochemically Assisted Deposition of Transparent, Mechanically Robust $\mathrm{TiO}_{2}$ Films for Advanced Applications. J. Nanopart. Res. 2013, 15 (11), 2087.

(17) Chang, G.; Zhang, J.; Oyama, M.; Hirao, K. Silver-NanoparticleAttached Indium Tin Oxide Surfaces Fabricated by a Seed-Mediated Growth Approach. J. Phys. Chem. B 2005, 109 (3), 1204-1209.

(18) Ward Jones, S. E.; Campbell, F. W.; Baron, R.; Xiao, L.; Compton, R. G. Particle Size and Surface Coverage Effects in the Stripping Voltammetry of Silver Nanoparticles: Theory and Experiment. J. Phys. Chem. C 2008, 112 (46), 17820-17827.

(19) Giovanni, M.; Pumera, M. Size Dependant Electrochemical Behavior of Silver Nanoparticles with Sizes of 10, 20, 40, 80 and 107 Nm. Electroanalysis 2012, 24 (3), 615-617.
(20) Ivanova, O. S.; Zamborini, F. P. Size-Dependent Electrochemical Oxidation of Silver Nanoparticles. J. Am. Chem. Soc. 2010, 132 (1), 70-72.

(21) Kresse, G.; Joubert, D. From Ultrasoft Pseudopotentials to the Projector Augmented-Wave Method. Phys. Rev. B: Condens. Matter Mater. Phys. 1999, 59 (3), 1758-1775 and references therein.

(22) Perdew, J. P.; Burke, K.; Ernzerhof, M. Generalized Gradient Approximation Made Simple. Phys. Rev. Lett. 1996, 77 (18), 38653868.

(23) Morgan, B. J.; Watson, G. W. A DFT+U Description of Oxygen Vacancies at the $\mathrm{TiO}_{2}$ Rutile (110) Surface. Surf. Sci. 2007, 601 (21), 5034-5041.

(24) Cheng, H.; Selloni, A. Energetics and Diffusion of Intrinsic Surface and Subsurface Defects on Anatase $\mathrm{TiO}_{2}(101)$. J. Chem. Phys. 2009, 131 (5), 054703.

(25) Puigdollers, A. R.; Schlexer, P.; Pacchioni, G. Gold and Silver Clusters on $\mathrm{TiO}_{2}$ and $\mathrm{ZrO}_{2}$ (101) Surfaces: Role of Dispersion Forces. J. Phys. Chem. C 2015, 119 (27), 15381-15389.

(26) Zhao, Z. Theoretical Study of Pt Cocatalyst Loading on Anatase $\mathrm{TiO}_{2}$ (101) Surface: From Surface Doping to Interface Forming. $J$. Phys. Chem. C 2014, 118 (42), 24591-24602.

(27) Rimoldi, L.; Ambrosi, C.; Di Liberto, G.; Lo Presti, L.; Ceotto, M.; Oliva, C.; Meroni, D.; Cappelli, S.; Cappelletti, G.; Soliveri, G.; et al. Impregnation versus Bulk Synthesis: How the Synthetic Route Affects the Photocatalytic Efficiency of $\mathrm{Nb} / \mathrm{Ta}: \mathrm{N}$ Codoped $\mathrm{TiO}_{2}$ Nanomaterials. J. Phys. Chem. C 2015, 119 (42), 24104-24115.

(28) Perdew, J. P.; Wang, Y. Accurate and Simple Analytic Representation of the Electron-Gas Correlation Energy. Phys. Rev. B: Condens. Matter Mater. Phys. 1992, 45 (23), 13244-13249.

(29) Selloni, A. Crystal Growth: Anatase Shows Its Reactive Side. Nat. Mater. 2008, 7 (8), 613-615.

(30) Vittadini, A.; Selloni, A.; Rotzinger, F. P.; Grätzel, M. Structure and Energetics of Water Adsorbed at $\mathrm{TiO}_{2}$ Anatase (101) and (001) Surfaces. Phys. Rev. Lett. 1998, 81 (14), 2954-2957.

(31) Ceotto, M.; Lo Presti, L.; Cappelletti, G.; Meroni, D.; Spadavecchia, F.; Zecca, R.; Leoni, M.; Scardi, P.; Bianchi, C. L.; Ardizzone, S. About the Nitrogen Location in Nanocrystalline NDoped $\mathrm{TiO}_{2}$ : Combined DFT and EXAFS Approach. J. Phys. Chem. C 2012, 116 (2), 1764-1771.

(32) Liu, L.; Bassett, W. A. Compression of $\mathrm{Ag}$ and Phase Transformation of NaCl. J. Appl. Phys. 1973, 44 (4), 1475-1479.

(33) Sun, Y. Shape-Controlled Synthesis of Gold and Silver Nanoparticles. Science 2002, 298 (5601), 2176-2179.

(34) Bader, R. F. W. Atoms in Molecules: A Quantum Theory; Oxford University Press, 1990.

(35) Tang, W.; Sanville, E.; Henkelman, G. A Grid-Based Bader Analysis Algorithm without Lattice Bias. J. Phys.: Condens. Matter 2009, 21 (8), 084204 and references therein.

(36) Schlexer, P.; Ruiz Puigdollers, A.; Pacchioni, G. Tuning the Charge State of $\mathrm{Ag}$ and $\mathrm{Au}$ Atoms and Clusters Deposited on Oxide Surfaces by Doping: A DFT Study of the Adsorption Properties of Nitrogen- and Niobium-Doped $\mathrm{TiO}_{2}$ and $\mathrm{ZrO}_{2}$. Phys. Chem. Chem. Phys. 2015, 17 (34), 22342-22360.

(37) He, Y.; Dulub, O.; Cheng, H.; Selloni, A.; Diebold, U. Evidence for the Predominance of Subsurface Defects on Reduced Anatase $\mathrm{TiO}_{2}$ (101). Phys. Rev. Lett. 2009, 102 (10), 106105.

(38) Tilocca, A.; Selloni, A. Methanol Adsorption and Reactivity on Clean and Hydroxylated Anatase(101) Surfaces. J. Phys. Chem. B 2004, 108 (50), 19314-19319.

(39) Herman, G. S.; Sievers, M. R.; Gao, Y. Structure Determination of the Two-Domain $(1 \times 4)$ Anatase $\mathrm{TiO}_{2}(001)$. Phys. Rev. Lett. 2000, 84 (15), 3354-3357.

(40) Yang, H. G.; Sun, C. H.; Qiao, S. Z.; Zou, J.; Liu, G.; Smith, S. C.; Cheng, H. M.; Lu, G. Q. Anatase $\mathrm{TiO}_{2}$ Single Crystals with a Large Percentage of Reactive Facets. Nature 2008, 453 (7195), 638-641.

(41) Zilberberg, L.; Mitlin, S.; Shankar, H.; Asscher, M. Buffer Layer Assisted Growth of Ag Nanoparticles in Titania Thin Films. J. Phys. Chem. C 2015, 119 (52), 28979-28991. 
(42) Boskovic, I.; Mentus, S. V.; Pjescic, M. Electrochemical Behavior of an $\mathrm{Ag} / \mathrm{TiO}_{2}$ Composite Surfaces. Electrochim. Acta 2006, 51 (14), 2793-2799.

(43) Lo Presti, L.; Ceotto, M.; Spadavecchia, F.; Cappelletti, G.; Meroni, D.; Acres, R. G.; Ardizzone, S. Role of the Nitrogen Source in Determining Structure and Morphology of N-Doped Nanocrystalline $\mathrm{TiO}_{2}$. J. Phys. Chem. C 2014, 118 (9), 4797-4807.

(44) Ceotto, M.; Di Liberto, G.; Conte, R. Semiclassical "Divide-andConquer" Method for Spectroscopic Calculations of High Dimensional Molecular Systems. Phys. Rev. Lett. 2017, 119 (1), 10401. 